Vol.16, No. 60, July 2021, 776-788

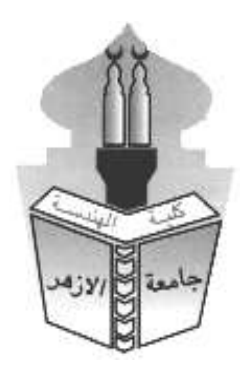

\title{
AUTOMATIC IDENTIFICATION AND EXTRACTION OF ADAPTIVE LEARNING OBJECTS FROM TRADITIONAL COURSEWARE
}

\author{
Mohammed Atef, Shehab Gamalel-Din, Gamal Tharwat \\ Systems \& Computers Dept, Faculty of Engineering, Al-Azhar University, Cairo, Egypt. \\ *Corresponding Author E-mail: mhmnbwy@gmail.com
}

\begin{abstract}
e-Learning is becoming the learning system of the future. However, many recent researches suggested replacing the "one size fits all" teaching model with the smarter model of adaptive learning in which the interpersonal differences between learners are considered the main shapers of lesson for a particular student.

However, the main hurdle for the adaptive model is building the repository of micro learning objects (MLOs) with reasonable sizes suitable for reassembling into lessons in a way that is more suitable for a particular student. The process of designing such MLOs is expensive and time-consuming, reasons with the least effect of dampening the model to the point that it may collapse.

Noteworthy, there is a wealth of digital learning contents and open-source educational curricula available online, but in the large granular traditional format. Therefore, this research proposes a Text processing intelligent framework for automatically identifying, extracting, and, annotating, those MLOs out of open digital video contents and PowerPoint courses, which are the most popular media types.
\end{abstract}

KEYWORDS: Applied computing $\rightarrow$ Education $\rightarrow$ e-Learning $\rightarrow$ Intelligent e-Learning.

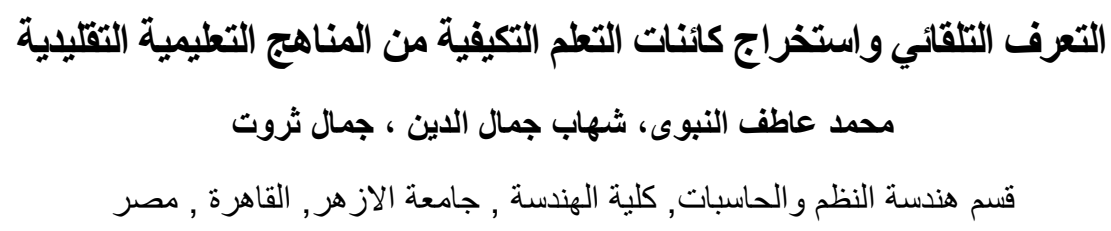

البريد الاليكتروني للباحث الرئيسى: E-mail mhmnbwy@gmail.com 


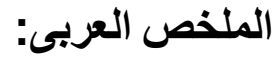

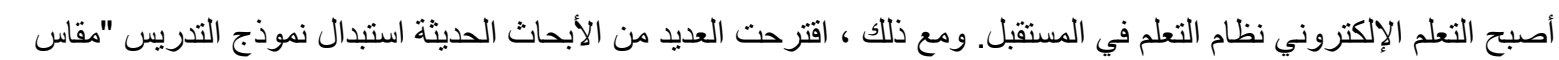

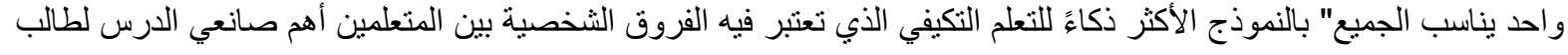

$$
\begin{aligned}
& \text { بأحجام معقولة مناسبة لإعادة التجميع MLOsومع ذلك ، فإن العقبة الرئيسية للنموذج التكيفي هي بناء مستودع كائنات التعلم الجزئي }
\end{aligned}
$$

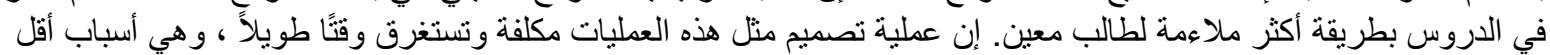

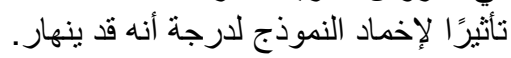

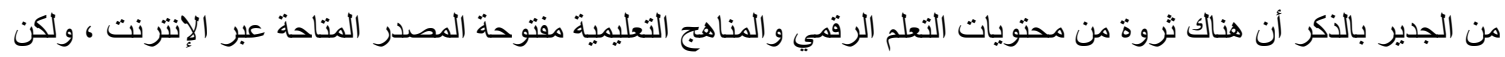

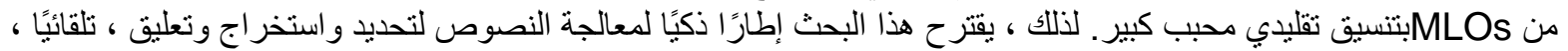

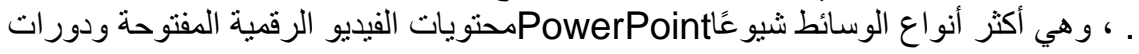

$$
\begin{aligned}
& \text { الكلمات المفتاحية : الحوسبة التطيقية, التطليم, التطليم الاككترونى, التعلم الإكتروني الأكي }
\end{aligned}
$$

\section{INTRODUCTION}

e-Learning is becoming the learning system of the future [1]. In fact, online learning has proven to be a great alternative to traditional methods of learning. However, most of the stakeholders of the education system have many concerns on the one-fits-all teaching model - the model that teaches all students similarly. Of course, human teachers can act better because they try to understand their student's psychology, background, objectives, etc. and adapt their teaching strategy accordingly. This can happen on small scales only, which is not human manageable when the number of students increase, hence, as small the class is as the better the adaptation occurs. This is what adaptive teaching or personalized learning are. Of course, one-to-one teaching that is usually achieved by private tutoring is considered the most effective learning system [2] —a system that cannot be achieved in almost all educational institutions, however, adaptive/personalized online learning can do unlimited scalable personalization according to the particular student's model [3]. Personalized e-Learning systems act like one-to-one private tutors who adapt their teaching style, strategy, and contents according to the learning context of the single learner .

The purpose of adaptive e-Learning systems is to enable learners to acquire knowledge and skills more efficiently (less time), more effectively (high learning outcomes), and more pleasantly. Hence, the adaptation process involves several constituents without them no adaptation can take place, among them are the student's model, the small granular LOs, and the concept ontology [4]. In this research, the focus is on the LOs .

Adaptive learning requires special types of Learning Objects (LOs) of small granularity, called Micro LOs (MLOs). A monolithic LO for a full lecture is not suitable for the personalization process [17]. Small granular MLOs, on the other hand, facilitate the reassembling process into personalized lessonsa process that reorganizes these MLOs differently for the different individual students [5], which explains the need for MLOs. Clearly, the manual splitting of a large full lecture into several smaller MLOs is not an easy process and requires special skills and intensive training. Hence, comes the idea of this research - the automatic identification and splitting of those MLOs out of a monolithic full lecture. Moreover, for the automatic lesson personalization process to work properly, it requires those MLOs to be properly selected according to specific criteria, which requires them to be annotated with descriptive metadata attributes that will be used as a base for the appropriate selection. Examples of such metadata attributes are Concept, Instructional Role, Technical format, and Language .

In summary, the objective of this research is to automate the process of identifying, extracting, and annotating those MLOs from a large full lecture, hence, splitting the lecture into its constituent MLOs covering a specific concept or a certain instructional role in a lesson, e.g., introduction, theory proving, example, or experiment, etc. In order to achieve this objective, this research employed different Text 
processing techniques. The target learning documents in this research are those that are most commonly used in education, namely, videos and PowerPoint presentations .

The remaining parts of this article are organized as follows. Section 2 reviews some related work, while Section 3 sheds light on the concept of Adaptive e-Learning. Section 4 gives an overview on the proposed model of extracting MLOs from PowerPoint presentations and Lecture Video files, while the implementation details for this model are presented in Section 5. Finally, Section 6 concludes.

\section{Related Work}

Several works have been proposed on previous years dealing with Multimedia Learning Object (MLO) Segmentation, Extraction and Annotation. These works can be categorized based on their segmentation strategy that can be classified into text-based segmentation or motion- based segmentation.

For instance, In [6], the authors used text-based segmentation, where they segmented the instructional videos using text-based segmentation on the basis of associated transcribed text. They used a Sliding window algorithm to detect topic boundaries by moving a sliding window of a certain size (e.g., six sentences) across the entire transcript by certain interval then compare the similarities between two neighboring windows of text, the places where similarities have a large variation are identified as potential topic boundaries. In [7], the authors detected topic changes by analyzing the co-occurrence of terms between transcript phrases based on word weights and term frequency, known as Inverse Document Frequency (TFIDF) NLP technique. In [8], the authors employed a linguistic-based approach for automatic video segmentation using Wikipedia texts. They proposed an algorithm to determine segment boundaries by matching transcript file blocks and Wikipedia texts with lecture video topics. The authors of [9] used a state-of-the-art word embedding text representation into their proposed method to segment videos in meaningful parts making use of the produced speech transcripts of a video .

In the above works on video lectures fragmentation required a transcript file of the video, some other works used different techniques like OCR (Optical Character Recognition) and ASR (Automatic Speech Recognition) to extract text from a video lecture, then use the extracted text in the segmentation process. For instance, [10] proposed a technique for segmenting lecture videos by analyzing its supplementary synchronized PowerPoint slides using an OCR. Similarly, [11] used slide transition recognition, text localization, and OCR techniques in order to determine fragment boundaries.

Noteworthy, the above-mentioned research used a text-based segmentation strategy for the segmentation process; however, another category of research used a motion-based strategy for segmenting video lectures. For instance, in [12], video is segmented into various scenes by identifying the transition of frames by the analysis of color histogram of lecture video's frames. In [13] They proposed an automatic lecture video indexing framework that compares lecture slide images with candidate video frames using Boosted deep neural networks. In [14], a supervised method is proposed using visual features along with transcripts in which SVM is trained on a lecture video to detect changes in events, e.g. "speaker writing on the blackboard" or "slide presentation. Fragment boundaries were extracted from these occurrences.

On the other hand, few research works on segmentation, extraction, and annotation for adaptive learning purposes. For instance, [15] presented an approach to migrating legacy video lectures into digital learning objects. The method detects slide transitions and extracts information from a presentation document (author name, title, date of creation) to obtain slide images, fill in the metadata of the learning object, and extract the table of contents from the presentation. The authors in [16] proposed a framework for the development of effective multi-media learning objects. They presented this framework to integrate with LMSs (Learning Management System) for the creation, storage, distribution, and evaluation of automatically extracted learning objects from digital media. They also extracted the relevant basic information from the media such as (keywords, title, and type). 


\section{Adaptive Learning}

The personalization process in adaptive e-Learning depends on a knowledgebase of two main constituents, namely the Learning Object Repository (LOR) and the Concept Ontology (CO). LORs and COs are incrementally built out of manipulating a lesson after another. This section sheds lights on those two constituents of the knowledgebase.

\subsection{Learning Objects}

In e-Learning, learning materials consist of multimedia learning objects referred to as learning objects (LOs). A Learning Object (LO) is a self-contained piece of learning contents. Those Learning objects are drawn from repositories (LOR) that are specified using standard metadata formats, such as SCORM [17] (Shareable Content Object Reference Model) or IEEE LOM [1]. Reusability is a major advantage of using learning objects, especially MLOs that are designed to present a certain piece of information regarding certain pedagogical aspect of a concept, e.g., an experiment, a theory proofing, an example of an application, etc.

LOs must be designed independently of a specific lesson, although can be extracted from a full lecture. Therefore, the same MLO can be shared among different lessons and contexts as long as it fits the required aspect in the new context. However, the selection of the most appropriate fitting MLO from a large LOR of millions of MLOs, might be a nightmare if done manually. Should the instructor review all the MLOs to select one, of course no. Every MLO must be properly specified by a set of metadata attributes that will be used to identify the appropriate MLOs [18]. There are several standards for metadata representations that have been developed to support both information exchange and resource discovery, e.g., [1] suggested a set of metadata attributes useful for adaptive and personalization purposes, as shown in Table 1. Some of these attributes are adopted in our research, such as Instructional role, format, and concept.

Table 1 LO's Metadata

\begin{tabular}{|c|c|c|}
\hline Fields & Attributes & Domain of Values \\
\hline \multirow{5}{*}{ Educational } & Instructional Role & $\begin{array}{r}\text { \{Introduction, Summary, Conclusion, Explanation, } \\
\text { Experiment, KeyPoints, Example- Exercise- undefined }\}\end{array}$ \\
\hline & Format & \{Figure, Table, Text, Graph, Image, Video Clip, Audio Clip\}. \\
\hline & Content Type & $\{$ Concrete, Abstract $\}$ \\
\hline & Depth type & \{General overview, In-depth\} \\
\hline & Concept & The Concept discussed by this LO. \\
\hline
\end{tabular}

\subsection{Concept Ontology}

A concept ontology is a network of semantically linked nodes representing knowledge concepts in a particular learning or instructional domain. An ontology helps individuals to share a common understanding of the subject domain. A large ontology is a composition of a collection of primitive ontologies for an entire domain 19 . [

The implementation of the ontology model is expected to improve the e-learning system's ability to obtain and process such learning materials in such a way that they can effectively facilitate personalization of learning in order to increase the quality of learning. Figure 1 shows an abstract example of a concept ontology. The most important semantic link is "Pats-Of" relationship that specifies the decomposition of a topic to its basic constituting subtopics. This is the one of focus in this research. 


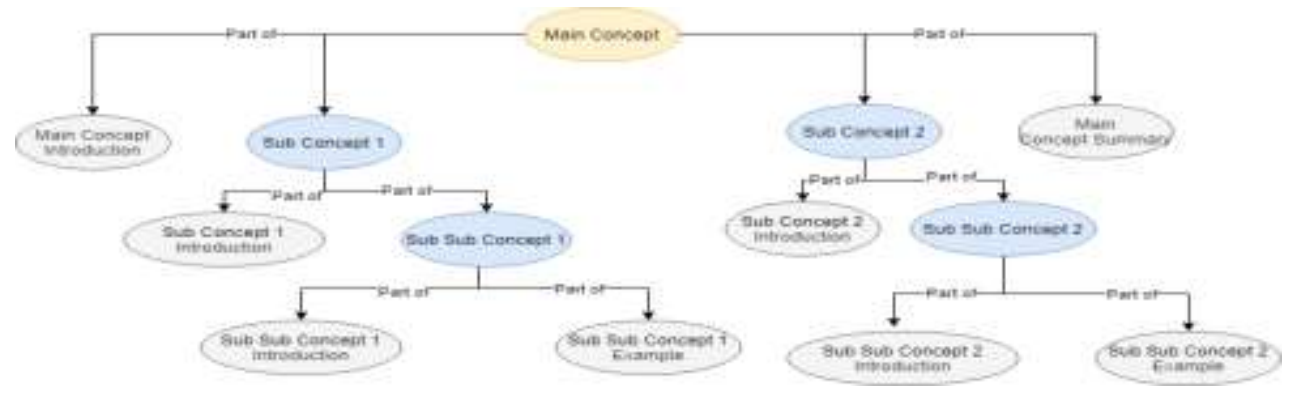

Figure 1. An Abstract example of a concept ontology.

\section{THE PROPOSED MODEL}

The objective of this research is to provide aids for the instructors in extracting both constituents of the adaptive knowledgebase, namely, LOs and partial Concept Ontology. Instructors are used in designing full lectures explaining a topic. This is usually done in one of the two forms of a video or a PowerPoint representation. The instructor does his/her lecture design according to his/her own preferences. Of course, it would be much unusual task to him to think in pieces instead of thinking holistically. Therefore, this research takes a full lecture and tries to split it into its constituent components of MLOs each is concerned about a single instructional role of the topic. Identifying the MLO should also be complemented by identifying its metadata attributes. On another dimension, the research addresses the extraction of the partial concept ontology as covered by the lecture. The annotated attributes are saved in the appropriate LOR while the partial ontology is integrated to the domain knowledge ontology.

Figure 2 draws the architecture block diagram demonstrating the different components of the LO extractors and their interconnections. The architecture consists of three main components. The first is the Multimedia Learning Object Extractor. This component is responsible for automatic extraction of Micro LOs from a given lecture video (VLO) or PowerPoint presentation (PPT LO). It takes a video (VLO) or PowerPoint Presentation (PPT LO) as an input to extract the different MLOs that are then analyzed and annotated with the appropriate metadata by the second component - the LO analyzer. The LOs are then stored in the MLO repository along with their metadata information stored in XML (Extensible Markup Language) format. The third component is responsible for incrementally building the knowledge Ontology related to the extracted LOs. It is worth noting that both the micro-LOR and the Knowledge Ontology are incrementally expanded each time a new LO is added to the repository. 


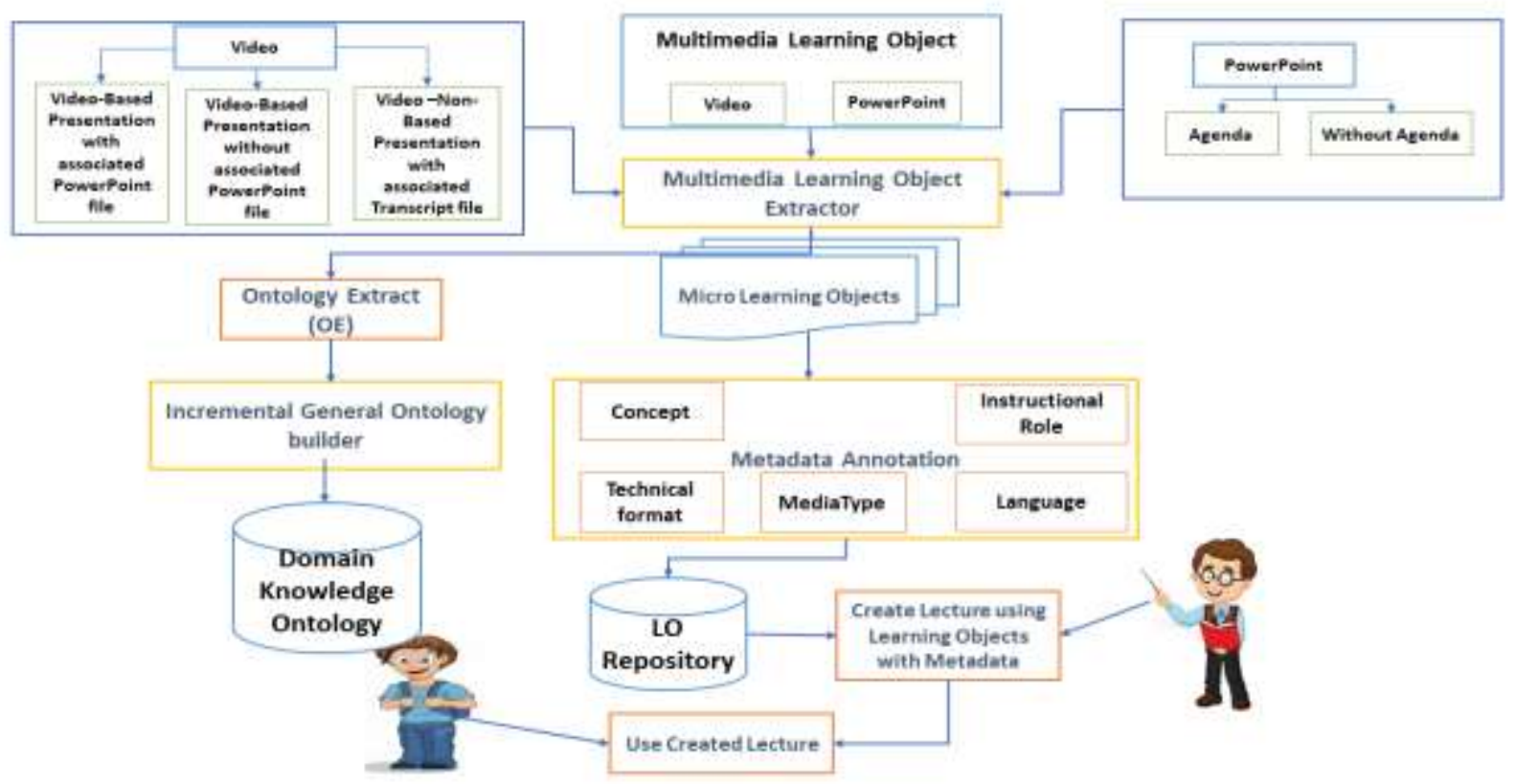

Figure 2. High-level architecture of the Micro LO Extractor prototypes.

\section{IMPLEMENTATION}

In this section, we shed lights on the algorithms that are used so far in identifying the micro LOs. Noteworthy, in this research, a prototype was implemented by python using several libraries as indicated in the description below.

\subsection{Extracting LOs from PowerPoint Presentations}

Normally PowerPoint presentations (PPTs) have no standard structure. For instance, one presentation may contain agenda with only main topics, while another may contain a more detailed agenda with subtopics, yet another may contain no agenda at all, similarly, all other constituting parts of the presentation. Therefore, a recommended standard template is designed, as shown in Figure 3. This standard structure is used as the basis for the transformation and processing, as depicted in Figure 4. If the PPT presentation is provided in the standard format at the first place, then it goes directly to the Standard PPT LO Extractor. Figure 5 demonstrates the LO extraction algorithm of the Standard PPT LO extractor. 


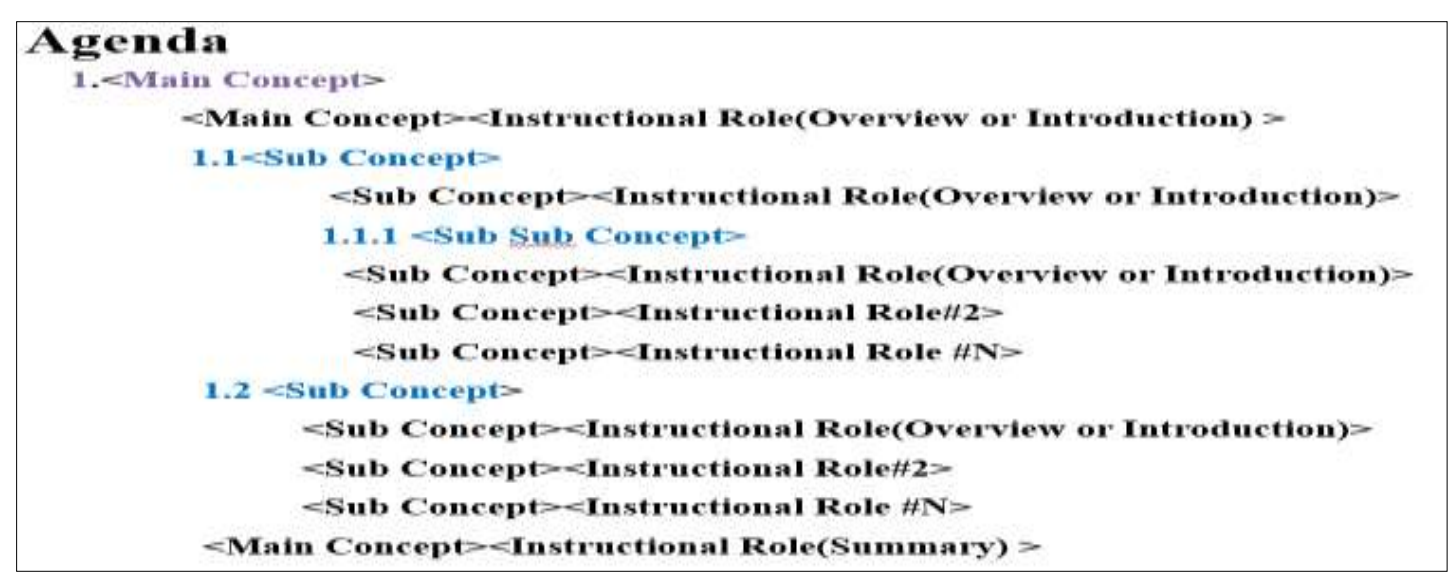

Figure 3. The Recommended Standard Structure for a Lecture in PowerPoint Presentation format.

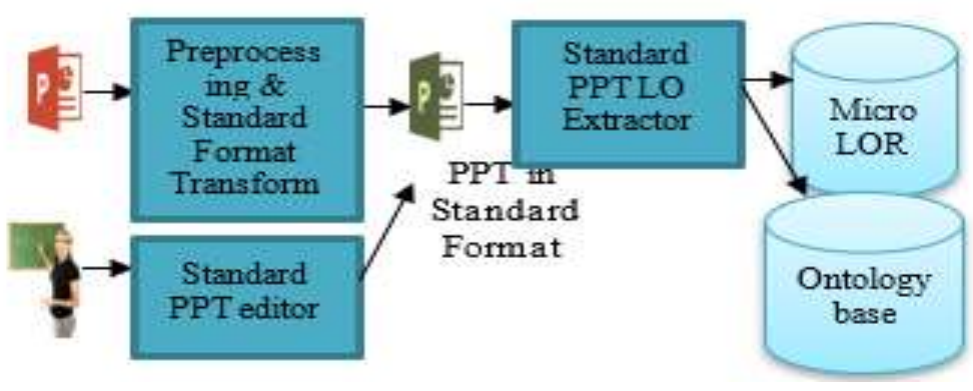

Figure 4. The process of Micro LO Generation.

\section{1- Preprocessing}

1.1. Extracting Presentation Title

1.2. Agenda Preprocessing

1.2.1. Identify Agenda Slide

1.2.2. Parsing

a. Agenda slide content

b. Other PowerPoint slides

1.2.3. Mapping Agenda content to PowerPoint slides

2- Generate Recommended Standard Format

3- Building Ontology

4- Extracting

3.1 Learning Objects

3.2 Learning Objects Metadata

Figure 5. The Algorithm of the Standard PPT LO. 


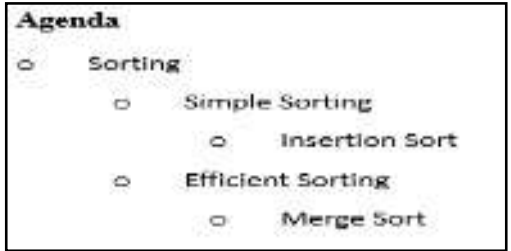

(a) Agenda before Preprocessing.

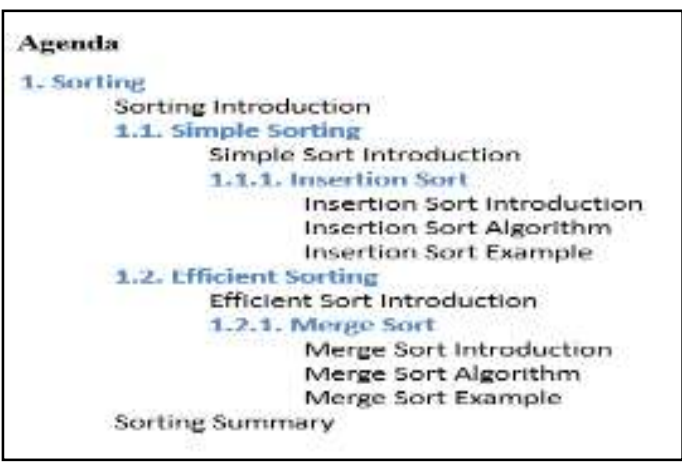

(b) Agenda after Preprocessing.

Figure 8. Sample Agenda Before and After

\section{PPT Pre-Processing}

The four steps of the algorithm are explained in what follows.

\section{a. Identify Agenda slide}

The starting point of the preprocessing process is to identify the Agenda slide. All the consecutive steps are guided by the Agenda. The title of the first few slides of the presentation are parsed for a synonym of the word ", such as "Topics", "outlines", "Road Map", "menu", ...etc. If not found in the title, the top part of the body of the slide is parsed for the same synonyms. Once identified, the Agenda slide is parsed and transformed into the standard format as follows.

\section{b. Parsing Agenda slide content}

Regular expression rules as shown in Figure 6 are employed on each item of the identified Agenda slide. Items matching these rules are identified as "Concepts", while the other items are identified as "Learning Objects". These rules are applied recursively on each identified "Concept" to determine its level (Concept or sub-concept or sub-sub-concept), an information that is later used in building the hierarchical Ontology of the lesson. For Presentations not following the recommended standard format, preprocessing takes place to transform the Agenda slide onto the standard format, see the algorithm outlined in Figure 7. Figure 8 depicts an Agenda example before and after preprocessing.

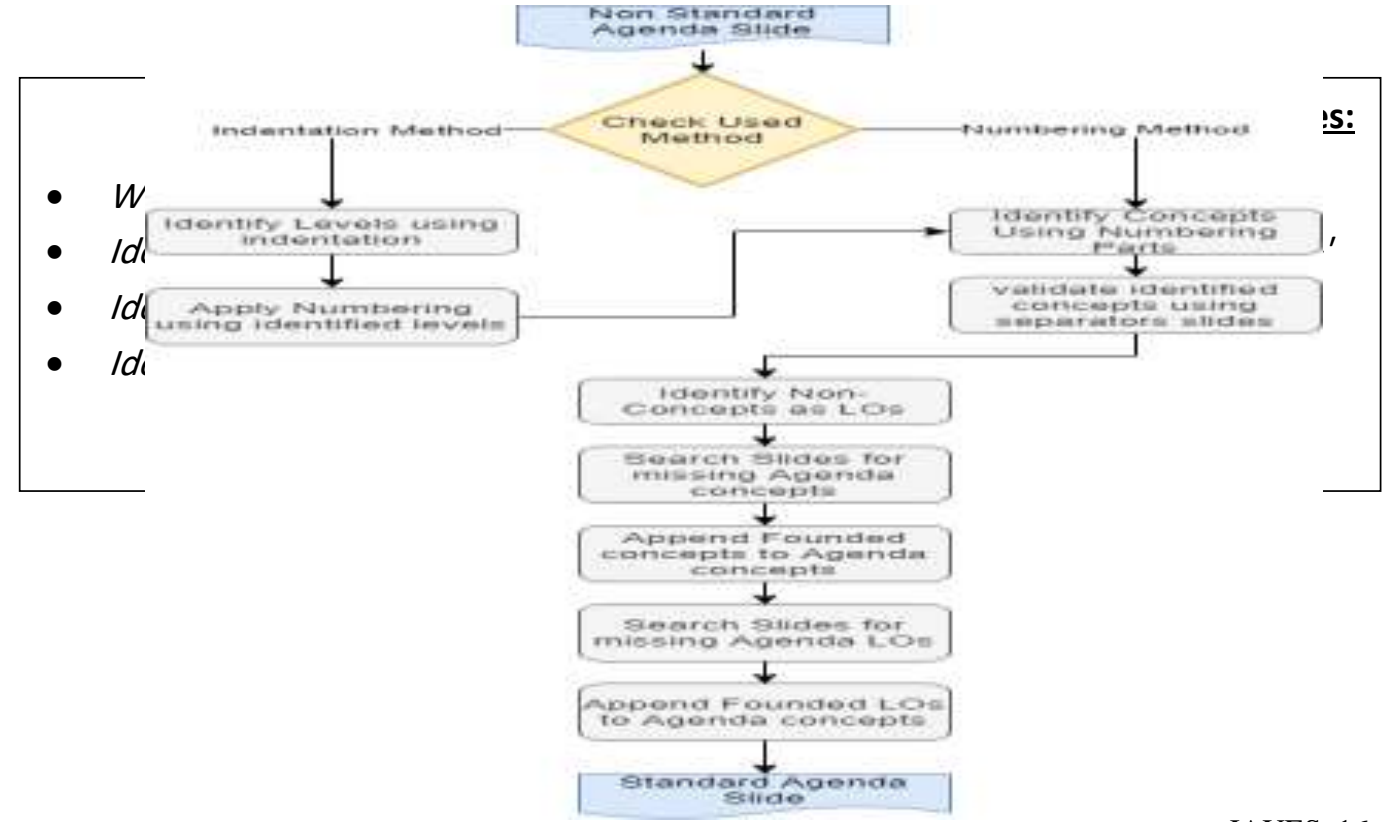

Figure 7. Preprocessing of Nonstandard Agenda slide. 


\section{c. Mapping Agenda Content to PowerPoint Slides}

After generating the standard "Agenda" slide that reflects the hierarchical structure of the lecture, comes the step of parsing the rest of the presentation slides. The Agenda "Concepts" are now searched for in the remaining slides using both the slides' titles and the separator slides, if any, by measuring the similarity between them. If the similarity is above a predefined threshold, then the matched slide is assigned to that concept; but if not, then a separator with concept title is added to standard target PowerPoint presentation.

d. Generating Standard Target Presentation

After preprocessing the Agenda content and Mapping Agenda Content to PowerPoint Slides and adjust PowerPoint slides a PowerPoint is transformed into the Recommended standard format and a new standard format presentation is generated; the format that is ten passed to the Standard LO Extractor to extract the micro LOs and store them in the LO Repository. Those LO are annotated with the appropriate metadata as defined by the Agenda most importantly, both the concept and the instructional role, as shown in Figure 9.

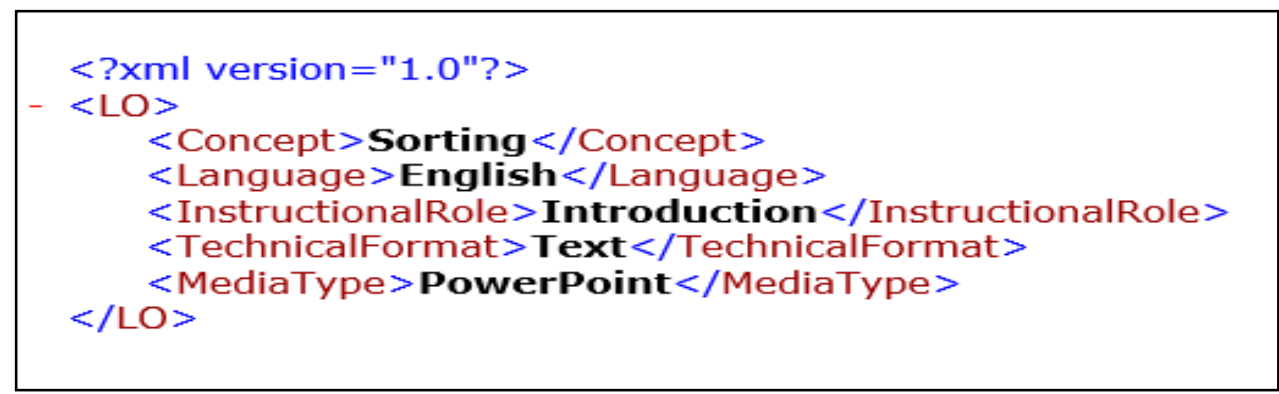

Figure 9. An Example of the Metadata of an Automatically Extracted LO.

Incremental Building of the Partial Concept Knowledge Ontology

The hierarchical ontology is generated with the aid of the hierarchy of the generated standard Agenda. This is done by linking each sub concept to its parent main concept, and by linking each sub-sub-concept to its parent sub-concept, and finally, by linking each LO to the parent concept. Figure 10 shows an extracted ontology. 


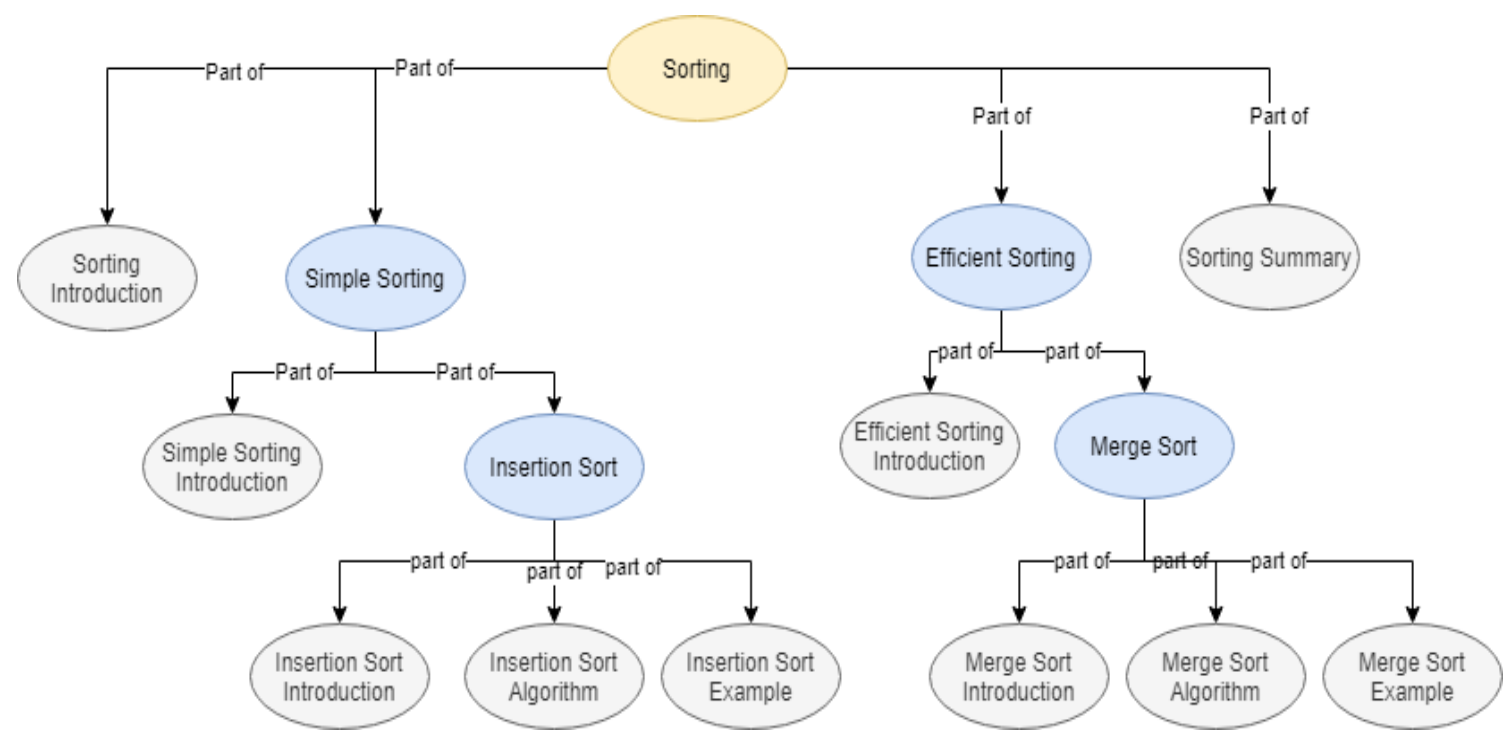

Figure 10. An Example of the Ontology Model Extracted by the Proposed Algorithm.

\subsection{Extracting Learning Objects from Video-Based Lectures with an Associated PowerPoint} In this section, we present an overview of the approach we followed for extracting Micro LOs from lecture videos-based presentations with associated PowerPoint file, the pipeline of which is illustrated in Figure 11.

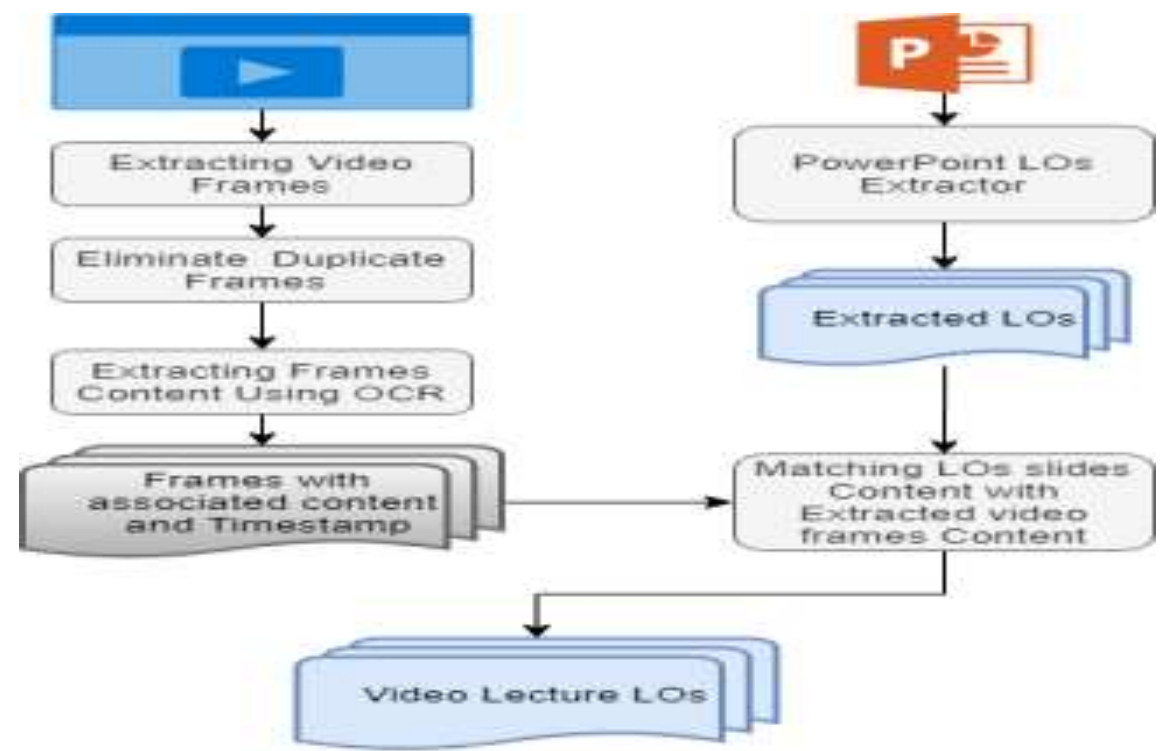

Figure 11. The Proposed Process of Extracting Video LOs from a Video-Based Presentation with Associated PowerPoint File. 
The proposed approach consists of six components. The first component is PowerPoint Learning Object Extractor, this component is responsible for automatic extraction of Micro LOs from a given PowerPoint presentation (PPTLO) as illustrated in section 5.1.

The second component is Video frames extractor, this component is responsible for extracting video frames using Open CV library [20], similar slides in video based presentation usually last for few seconds to minutes. So, only one frame per second (fps) is considered to minimize the count of extracted video frames.

Then duplicate consecutive frames are eliminated in third component by computing frame-to-frame difference and compared this value with predefined threshold if value is greater than predefined threshold then second frame is eliminated.

In fourth component we extract remaining video frames content by applying OCR, For reaching high accuracy in extracting text from video frames a Google Tesseract OCR [21] is used in this research. After applying OCR, Frames with the same content are eliminated by removing all these frames except the first frame. In this implementation we have used python Google Tesseract OCR library.

Since cosine similarity is often used to measure document similarity in text analysis $[22,23,24]$. The cosine Similarity value is bound by a constrained range from 0 and 1 where the value which is closer to 1 is considered as more similar, and vice versa. Therefore, the last component to describe is Matching LOs slides Content with Extracted video frames Content, in which we match the content in remaining video frames to the content in the extracted LOs slides from PowerPoint Learning Object Extractor by measuring Cosine Similarity in a vector space model between each LOs slide content and each extracted video frames content. In order to relate the PowerPoint slide to its corresponding frame in the video, we need to measure the similarities between the occurrence frequency of all terms in the text of a slide and those which are extracted from the video frames content. Therefore, we transform a given slide text into a vector on the basis of the frequency (count) of each word that occurs in the entire text then we create a matrix in which each unique word is represented by a column of the matrix, and each text sample from the slide content is a row in the matrix. Then we calculate Cosine similarity between rows in created matrix. After calculating Cosine similarity, LOs Slides are matched with video frames using highest similarity value between each LO slides and Video Frames. Finally, video LOs are generated by applying segmentation on input video using matched frames time stamps. In this implementation we have used python Scikit-learn [25] library.

\section{CONCLUSION}

Lecture Video and PowerPoint presentations are the most multimedia commonly used in e- learning. Extracting Micro LOs from this multimedia are becoming necessary for personalized/adaptive learning. In this article, we presented a model and a framework for identifying and annotating micro LOs with different meta-data from PowerPoint presentations in standard and nonstandard formats as well as from video-based lectures using NLP and text processing techniques.

More work is planned for future research. In this stage of research, video lectures are manipulated assuming that they are centered on associated PPT slides that the instructor uses all over the lecture in his/her explanation. For future research, we plan to relax this condition and investigate how to manipulate a video lecture independently of an associated PPT. 
AUTOMATIC IDENTIFICATION AND EXTRACTION OF ADAPTIVE LEARNING OBJECTS FROM TRADITIONAL COURSEWARE

\section{ACKNOWLEDGMENT}

This research is partially supported by the Egyptian Information Technology Industry Development Authority (ITIDA) under ITAC project number CFP159 titled "EduEdges: An Adaptive e-Learning Platform".

\section{REFERENCES}

1. S. Gamalel-din, "Smart E-Learning School of the Future : Project Report," no. May, 2015.

2. A. LW et al., A Taxonomy for Learning, Teaching, and Assessing: A Revision of Bloom's Taxonomy of Educational Objectives. 2001.

3. S. A. Gamalel-din, "LEARNING STYLES, LEARNING OBJECTS , AND ADAPTIVE ELEARNING."

4. S. A. Gamalel-Din, "An Intelligent Etutor-Student Adaptive Interaction Framework," in Proceedings of the 13th International Conference on Interacción Persona-Ordenador, 2012, doi: 10.1145/2379636.2379648.

5. R. Al-Otaibi and S. Gamalel-Din, "Intelligent querying for adaptive course preparation and delivery in e-learning," Proc. 8th IASTED Int. Conf. Web-Based Educ. WBE 2010, no. Wbe, pp. 12-21, 2010, doi: 10.2316/p.2010.688-026.

6. M. Lin, "for Lecture Videos : A Linguistics-Based Approach," Int. J., vol. 1, no. June, pp. $27-$ 45, 2005.

7. M. Almousa, R. Benlamri, and R. Khoury, "NLP-Enriched Automatic Video Segmentation," Int. Conf. Multimed. Comput. Syst. -Proceedings, vol. 2018-May, pp. 1-6, 2018, doi: 10.1109/ICMCS.2018.8525880.

8. R. R. Shah, Y. Yu, A. D. Shaikh, and R. Zimmermann, "TRACE: Linguistic-Based Approach for Automatic Lecture Video Segmentation Leveraging Wikipedia Texts," Proc. - 2015 IEEE Int. Symp. Multimedia, ISM 2015, pp. 217-220, 2016, doi: 10.1109/ISM.2015.18.

9. A. Leibetseder, S. Kletz, and K. Schoeffmann, ECAT - Endoscopic Concept, vol. 2. Springer International Publishing, 2019.

10. X. Che, H. Yang, and C. Meinel, "Lecture video segmentation by automatically analyzing the synchronized slides," MM 2013 - Proc. 2013 ACM Multimed. Conf., pp. 345-348, 2013, doi: $10.1145 / 2502081.2508115$.

11. H. Yang, M. Siebert, P. Lühne, H. Sack, and C. Meinel, "Automatic lecture video indexing using video OCR technology," Proc. - 2011 IEEE Int. Multimedia, ISM 2011, no. December 2011, pp. 111-116, 2011, doi: 10.1109/ISM.2011.26.

12. D. Ma and G. Agam, "Lecture video segmentation and indexing," Doc. Recognit. Retr. XIX, vol. 8297, p. 82970V, 2012, doi: 10.1117/12.910594.

13. D. Ma, X. Zhang, X. Ouyang, and G. Agam, "Lecture video indexing using boosted margin maximizing neural networks," Proc. - 16th IEEE Int. Conf. Mach. Learn. Appl. ICMLA 2017, vol. 2017-Decem, pp. 221-227, 2017, doi: 10.1109/ICMLA.2017.0-155. 
14. C. Bhatt et al., "Multi-factor segmentation for topic visualization and recommendation: The MUST-VIS system," MM 2013 - Proc. 2013 ACM Multimed. Conf., pp. 365-368, 2013, doi: $10.1145 / 2502081.2508120$.

15. A. De Lucia, R. Francese, M. Giordano, I. Passero, and G. Tortora, "Migrating legacy video lectures to multimedia learning objects," ICEIS 2006 - 8th Int. Conf. Enterp. Inf. Syst. Proc., vol. SAIC, pp. 51-58, 2006.

16. A. S. Imran and F. A. Cheikh, "Multimedia learning objects framework for e-learning," 2012 Int. Conf. E-Learning E-Technologies Educ. ICEEE 2012, pp. 105-109, 2012, doi: 10.1109/ICeLeTE.2012.6333417.

17. O. Bohl, D. Schellhase, R. Sengler, P. Dr, and U. Winand, "The sharable content object reference model (SCORM) - A critical review,” Proc. Int. Conf. Comput. Educ., May 2004.

18. H. S. Al-Khalifa and H. C. Davis, "The evolution of metadata from standards to semantics in elearning applications," Proc. Seventeenth ACM Conf. Hypertext Hypermedia, HT'06, vol. 2006, no. February 2014, pp. 69-72, 2006, doi: 10.1145/1149941.1149956.

19. N. Guarino, "Understanding, building and using ontologies," Int. J. Hum. Comput. Stud., vol. 46, no. 2-3, pp. 293-310, 1997, doi: 10.1006/ijhc.1996.0091.

20. [20] G. Bradski, “The openCV library,” Dr. Dobb’s J. Softw. Tools, vol. 25, Jan. 2000.

21. R. Smith, An Overview of the Tesseract OCR Engine, vol. 2. 2007.

22. D. Gunawan, C. A. Sembiring, and M. A. Budiman, "The Implementation of Cosine Similarity to Calculate Text Relevance between Two Documents," J. Phys. Conf. Ser., vol. 978, no. 1, 2018

23. L. Zahrotun, "Comparison Jaccard similarity, Cosine Similarity and Combined Both of the Data Clustering With Shared Nearest Neighbor Method,” Comput. Eng. Appl. J., vol. 5, no. 1, pp. $11-18,2016$

24. P. Sitikhu, K. Pahi, P. Thapa, and S. Shakya, "A comparison of semantic similarity methods for maximum human interpretability," arXiv, 2019.

25. F. Pedregosa et al., "Scikit-learn: Machine Learning in Python," J. Mach. Learn. Res., vol. 12, no. 85, pp. 2825-2830, 2011. 